\title{
Assimetrias manuais e complexidade da tarefa em habilidades de apontamento
}

\author{
Guilherme M. Lage ${ }^{1,2}$ \\ Lívia G. Gallo ${ }^{1}$ \\ Maíra G. de Miranda ${ }^{1}$ \\ Danilo R. Vieira ${ }^{1}$ \\ David J. Schickler ${ }^{1}$ \\ Robledo R. Coelho ${ }^{2}$ \\ Herbert Ugrinowitsch ${ }^{3}$ \\ Rodolfo N. Benda ${ }^{3}$
}

https://doi.org/10.5628/rpcd.08.01.47

\author{
${ }^{1}$ Faculdade de Ciências da Saúde (FCS) \\ Universidade FUMEC \\ Brasil \\ ${ }^{2}$ Laboratório do Comportamento Humano (LACOH) \\ Faculdades Unidas do Norte de Minas \\ Brasil \\ ${ }^{3}$ Grupo de Estudo em Desenvolvimento e \\ Aprendizagem Motora (GEDAM) \\ Universidade Federal de Minas Gerais \\ Brasil
}

\section{RESUMO}

O presente estudo investigou as assimetrias manuais em duas tarefas com níveis de complexidade distintos. Para tal, foram utilizadas duas tarefas de apontamento com diferentes números de componentes, definidas como tarefa de menor complexidade e tarefa de maior complexidade. Dez participantes destros executaram blocos de 5 tentativas de ambas as tarefas com a mão direita e a mão esquerda. Os movimentos foram filmados a uma taxa de 400 quadros/segundo. Os resultados indicaram um efeito para a complexidade da tarefa nas medidas de tempo de reacção, tempo de contacto para o $1^{\circ}$ alvo (tempo de movimento), pico de velocidade para o $1^{\circ}$ alvo e tempo proporcional para se atingir o pico de velocidade para o $1^{\circ}$ alvo. Foram encontradas assimetrias manuais em ambas as tarefas para as medidas de tempo de respostas e número de correcções após o pico de velocidade para o $1^{\circ}$ alvo. Dessa forma, não foi encontrado o efeito da complexidade sobre as assimetrias manuais em tarefas de apontamento. Os resultados são discutidos em relação à limitada generalização de prévios resultados de pesquisas comportamentais e neurofisiológicas.

\section{ABSTRACT}

Manual asymmetries and task complexity in aiming skills

This study investigated the manual asymmetries in two tasks with different levels of complexity. Two aiming tasks with different number of components were used. The first one was defined as discrete task (simple task) and the second one defined as serial task (complex task). Ten right-handed participants performed one block of 5 trials of both tasks with their right and left hands. The movements were recorded at a sample rate of $400 \mathrm{~Hz}$. The results indicated an effect of task complexity to the measures of reaction time, contact time to the $1^{\text {st }}$ target (movement time), peak velocity to the $1^{\text {st }}$ target, proportional time to reach the peak velocity to the $1^{\text {st }}$ target. Manual asymmetries were found in both tasks in the variables response time and number of corrections after the peak velocity to the $1^{\text {st }}$ target. The effects of task complexity on manual asymmetries were not observed. The results are discussed in relation to the restricted generalization of previous findings found in neurophysiological and behavioral studies.

Key-words: manual asymmetries, task complexity, aiming skills

Palavras-chave: assimetrias manuais, complexidade da tarefa, habilidades de apontamento 


\section{INTRODUÇÃO}

Um dos modelos mais frequentemente adoptado no estudo de habilidades manuais como as de apontamento é o modelo híbrido de duas fases de Woodworth (22), no qual o primeiro momento da acção, fase de impulso inicial, é controlado centralmente e tem como função deslocar de forma balística o membro em direcção ao alvo. Já próximo ao ponto final do movimento, tem início a segunda fase, controle corrente do movimento, na qual a informação aferente sobre as posições relativas do membro e alvo é utilizada para possíveis ajustes na trajectória do movimento para que o alvo seja alcançado com precisão(10).

As tarefas de apontamento estão certamente entre as mais utilizadas no estudo das assimetrias manuais de desempenho(6), e em sujeitos destros, a mão dominante não somente produz movimentos mais rápidos, mas também movimentos mais precisos e consistentes(3). A emergência dessas assimetrias encontra-se na interacção entre factores biológicos e ambientais. Focando nos aspectos biológicos, a especialização hemisférica apresenta uma função predominante nas assimetrias(16). Assume-se que o sistema hemisfério esquerdo/mão direita apresenta uma especialização tanto na capacidade de parametrizar valores de força, caracterizados pela magnitude e timing do movimento, quanto na utilização de feedback visual e no controle corrente do movimento(4). Por outro lado, há uma tendência da mão esquerda reagir mais rapidamente do que a mão direita(6). Nesse caso, o sistema hemisfério direito/mão esquerda apresenta uma especialização na parametrização espacial do movimento devido a geração de um mapeamento espacial entre a locação do alvo e a posição de um determinado efector ou efectores, o que reflecte em um menor tempo de preparação do movimento(5).

Contudo, o grau de assimetria manual de desempenho difere de tarefa para tarefa sendo influenciado, entre outras variáveis, pela complexidade da tarefa ${ }^{(2,}$ 18). É importante destacar a inexistência de uma definição explícita de "complexidade da tarefa" nos estudos sobre assimetria manual (8). Esta é uma questão de difícil solução, tendo em vista que depende da relativização do que é comparado entre as tarefas. Porém, esta questão não diminui a importância das investigações sobre a relação entre assimetrias manuais e complexidade da tarefa, tendo em vista que é possível identificar em quais características esta relação é mais bem observada. Como exemplo, características intrínsecas da tarefa como número de componentes envolvidos no movimento e características extrínsecas como a localização espacial do movimento são fatores apontados como de interferência na complexidade da tarefa(2). Essas diferenças sobre a acepção de complexidade da tarefa podem explicar em parte os resultados inconsistentes encontrados na literatura. Enquanto alguns resultados sugerem certa relação linear positiva entre complexidade da tarefa e assimetria de desempenho( 7,15$)$, outros indicam uma relação contrária entre nível de complexidade e assimetria ${ }^{(8)}$ como descrito abaixo. Flowers(7) e Provins e Magliaro(15) investigaram a relação entre os níveis de assimetria manual e complexidade através da comparação de medidas de desempenho em diferentes tarefas (ex. tarefa de apontamento versus tarefa de toques repetidos). Todavia, essa comparação pode ser sem efeito, pois a assimetria manual não somente depende da complexidade das tarefas, mas também da natureza das mesmas e do nível de experiência do executante em cada tarefa(2). Seguindo essa linha de raciocínio, seria importante testar a complexidade da tarefa em tarefas de mesma natureza, questão que retomaremos adiante.

A relação entre níveis de assimetria manual e complexidade encontrada por Hausmann, Kirk e Corballis(8) tem suporte nos estudos com análise neurofisiológica $(17,19,21)$. Em estudos que utilizaram neuroimagem observa-se que movimentos menos complexos promovem baixa activação cortical ipsilateral, enquanto movimentos mais complexos levam a uma maior activação bi-hemisférica(21). Os resultados sugerem que uma maior participação do hemisfério ipsilateral no controle do movimento atenue a assimetria de desempenho entre as mãos.

Um ponto que merece destaque é que pouca atenção tem sido despendida no sentido de se estudar a relação entre assimetria manual e complexidade da tarefa em estudos que utilizam medidas comportamentais, além de ter sido raramente investigada de forma isolada(2). Uma possível razão para esse quadro está em parte associada à dificuldade em definir de forma sistemática complexidade da tarefa, e em parte pelas 
análises indirectas da complexidade, já que o objectivo principal de vários destes estudos não contempla prioritariamente esta questão. Podemos citar como uma das poucas excepções o estudo de Hausmann et al.(8), no qual foi manipulada a complexidade da tarefa em habilidades de toques contínuos como os dedos. Apesar das tarefas de apontamento serem certamente as mais utilizadas nos estudos sobre assimetria manual(6), pouca atenção tem sido dada a manipulação da complexidade nesse tipo de tarefa. Habilidades de apontamento com diferente número de componentes são importantes para testar essa questão, pois apresentam natureza semelhante e podem ter complexidade distinta.

Um tema relacionado à complexidade da tarefa é o efeito do one-target advantage (OTA) (1,11). O OTA é observado através da comparação do tempo de movimento de uma tarefa com apenas um componente, tarefa simples, e o primeiro componente de uma tarefa de dois ou mais componente, tarefa complexa, tendo ambos os componentes a mesma distância. O tempo de movimento da tarefa simples é menor do que o tempo de movimento do primeiro componente da tarefa que exige a implementação de um segundo componente $^{(1)}$. O efeito da complexidade da tarefa sobre medidas comportamentais foi inicialmente observado por Henry e Rogers ${ }^{(9)}$, tendo os mesmos argumentado que o número de elementos constituintes de um determinado tipo de movimento é a principal característica da complexidade da tarefa. Um maior tempo para a preparação do movimento é observado em tarefas de maior complexidade comparadas a tarefas de menor complexidade. Neste caso, o último estágio do processamento, estágio da programação motora, demanda maior tempo para organizar um maior número de comandos motores. Lavrysen, Helsen, Tremblay, Elliott, Adam, Feys e Buekers ${ }^{(11)}$ combinaram duas variáveis, assimetria lateral e OTA, e encontraram o efeito do OTA em movimentos de apontamento realizados em abdução, partindo da região medial do corpo para a extremidade ipsilateral. A meta da tarefa foi realizar os movimentos em direcção a uma chave ( $1^{\circ}$ alvo) com maior velocidade e precisão possível. Os resultados da análise cinemática mostraram que comparado ao movimento de maior complexidade, o movimento de menor complexidade apresentou: 1) maior pico de velocidade, 2) maior variabilidade do pico de velocidade, 3) maior tempo para se atingir o pico de velocidade e 4) uma maior variabilidade na medida de tempo proporcional para se atingir o pico de velocidade. Através desses resultados, Lavrysen et al.(11) concluíram que tanto os processos envolvidos no planejamento do movimento, quanto os ajustes on-line via feedback contribuem para o OTA. Contudo, não foi encontrada diferença no número de descontinuidades no perfil de aceleração/desaceleração após o pico de velocidade, medida essa que melhor reflecte os ajustes on-line realizados via feedback. A alta exigência espacial da tarefa, observada na obrigatoriedade de tocar o alvo (chave) para registar o tempo de movimento, pode ter gerado estratégias específicas de controle, que pela análise dos resultados sugere ter sido mais de cunho central do que periférico. Além disso, os autores assumiram que o OTA é um fenômeno robusto que independe das assimetrias manuais. Entretanto, é possível que resultados distintos sejam encontrados em espaços de trabalho diferentes do investigado. As assimetrias manuais são influenciadas pelo espaço de trabalho devido às diferentes exigências geradas nos processos atencionais e/ou no processamento visuomotor reflectindo em diferentes níveis de activação bi-hemisférica(12, 20). O objectivo do presente estudo foi investigar as assimetrias manuais em tarefas com níveis de complexidade diferentes. Apoiados nos resultados de pesqui-

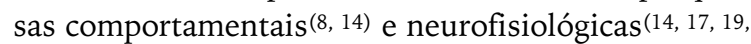
21), é possível levantar a hipótese de que menor nível de assimetria será encontrado na tarefa de maior complexidade. Uma proposta adicional foi a de investigar os efeitos do OTA e assimetria manual em movimentos realizados em um espaço de trabalho ainda não investigado, tendo em vista que esta variável influencia nos processos envolvidos na programação $\operatorname{motora}^{(12,20)}$.

\section{MÉTODO}

\section{Amostra}

Participaram do experimento 10 sujeitos universitários destros de ambos os sexos, índice médio de lateralidade (preferência manual) igual a $80^{(13)}$, na faixa etária entre 18 e 35 anos (média $=25,8 \pm 5,4$ anos), sendo todos voluntários e com consentimento livre e esclarecido. 


\section{Instrumentos e tarefa}

Para a determinação do índice de lateralidade, foi empregado o Inventário de Dominância Lateral de Edimburgo(13). Para a análise cinemática dos movimentos de apontamento foi utilizado o sistema Simi Motion 7.0, (Simi Reality Motion Systems GmbH). Duas câmaras de vídeo Basler de alta velocidade (400 quadros por segundo) foram utilizadas para a gravação dos vídeos. Um sistema de calibração (CELG Moldes LTDA) em forma de cubo com $20 \mathrm{~cm}$ de arresta foi utilizado. Foram utilizados dois diodos emissores de luz controlados por um software para o fornecimento do estímulo visual fornecido ao início de cada tentativa. Dois marcadores refletivos foram utilizados para identificar os pontos anatómicos de interesse, as falanges distais dos dedos indicadores direito e esquerdo. A tarefa consistiu em movimentos de apontamento caracterizados pelo deslocamento do membro superior a alvos pré-determinados sobre uma plataforma que deveriam ser contactados com o dedo indicador. A distância entre alvos foi de $20 \mathrm{~cm}$ de centro a centro e o diâmetro dos mesmos de 1,5 $\mathrm{cm}$, gerando assim um ID de 4.74 bits. Os alvos foram posicionados a $45^{\circ}$ à esquerda e à direita do ponto inicial do movimento (Figura 1).

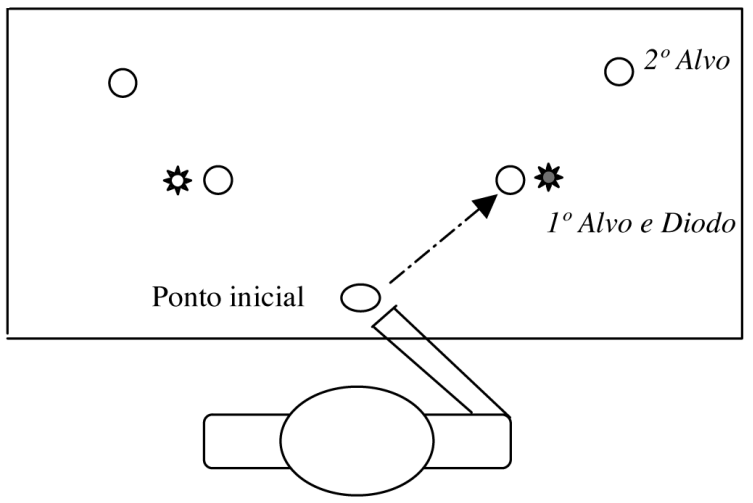

Figura 1. Exemplo de uma tentativa realizada com a meta de movimento simples (discreto] e execução com o membro direito.

\section{Delineamento experimental e procedimentos}

Inicialmente, sem a presença do participante, foi realizada a calibragem das dimensões espaciais para a análise tridimensional pelo software Simi Motion 7.0. Para a reconstrução 3D dos movimentos foi utilizado o protocolo DLT padrão. As câmeras foram posicionadas lateralmente à direita e à esquerda da mesa em que estavam dispostos os alvos circulares, em ângulos de aproximadamente $45^{\circ}$ ao ponto inicial do movimento a distância de 2 metros e altura de 150 centímetros. Todas as medidas utilizadas foram registadas através das filmagens. A colecta de dados foi realizada individualmente. Todos os participantes receberam demonstrações e instruções verbais padronizadas sobre o experimento. Após as instruções, foram afixados marcadores refletivos nas falanges distais dos dedos. Em seguida, o participante assentou em frente à mesa com os alvos e posicionou a cadeira de forma que a altura de seu tronco fosse confortável para a execução dos movimentos. A região medial do corpo ficou alinhada ao ponto inicial de movimento. Todos os sujeitos praticaram 3 tentativas de ambientação às metas e características da tarefa com ambas as mãos. Após a ambientação, teve início a colecta de dados. O participante era informado previamente sobre o membro a ser utilizado e quais alvos deveriam ser contactados em cada bloco de tentativas. Após a informação "prepara" fornecida pelo experimentador, o participante deveria repousar o dedo indicador pré-selecionado sobre o ponto inicial do movimento marcado na mesa. Um estímulo luminoso era emitido por um diodo disposto lateralmente a $2 \mathrm{~cm}$ do primeiro alvo a ser contactado, em um intervalo que variou aleatoriamente entre 3 e 5 segundos. O participante iniciava o movimento em direção ao alvo em maior velocidade e precisão possível. A tarefa de maior complexidade consistiu em posicionar o dedo indicador sobre o ponto inicial e contactar os alvos intermediário e final. A tarefa de menor complexidade consistiu em posicionar o dedo indicador sobre o ponto inicial e contactar apenas o $1^{\circ}$ alvo (Figura 1 ). Cada participante executou 5 tentativas consecutivas de cada tarefa com a mão esquerda e a mão direita. A ordem de execução das combinações tarefa versus mão foi contrabalançada entre os participantes.

\section{Medidas}

As variáveis dependentes de desempenho foram: 1) tempo de reação, 2) tempo de resposta e 3) tempo de contacto para o $1^{\circ}$ alvo. As variáveis dependentes cinemáticas foram: 1) pico de velocidade para o $1^{\circ}$ 
alvo, 2) tempo proporcional para se atingir o pico de velocidade para o $1^{\circ}$ alvo e 3 ) número de correcções após o pico de velocidade para o $1^{\circ}$ alvo.

Devido ao objectivo do presente estudo, não foram analisadas isoladamente as medidas de desempenho e as medidas cinemáticas do movimento para o $2^{\circ}$ alvo na tarefa de maior complexidade. Os tempos de resposta foram analisados separadamente em termos de complexidade. Este critério deve-se ao diferente número de componentes que compunham as tarefas. As medidas foram definidas da seguinte forma: o tempo de reacção foi definido como o período de tempo entre o $1^{\circ}$ quadro da filmagem em que o estímulo visual foi apresentado e o quadro que antecedeu o início do movimento. O início do movimento foi definido como o primeiro quadro no qual a velocidade absoluta excedeu o valor de $30 \mathrm{~mm} / \mathrm{s}$ e permaneceu dentro desse limite por um período maior que 10 quadros de amostragem. O fim do movimento foi definido como o primeiro quadro no qual a velocidade absoluta atingiu um valor abaixo de 30 $\mathrm{mm} / \mathrm{s}$ e permaneceu dentro desse limite por um período maior que 10 quadros. Os tempos de resposta foram definidos como: 1) a soma do tempo de reacção e tempo de movimento para o $1^{\circ}$ alvo (tarefa simples) e 2) a soma do tempo de reacção, do tempo de movimento para o $1^{\circ}$ alvo e do tempo de movimento para o $2^{\circ}$ alvo (tarefa complexa).

$\mathrm{O}$ intervalo de tempo entre o início do movimento e o $1^{\circ}$ quadro em que o dedo indicador contactou o alvo ou suas proximidades foi definido como tempo de contacto para o $1^{\circ}$ alvo. Esta medida foi adoptada para a comparação do tempo de movimento entre as tarefas de diferentes complexidades sem as correcções realizadas após o contacto do dedo indicador com o alvo. Isto se deve à diferente natureza das correcções quando se compara movimentos em que o dedo deve parar sobre o alvo (tarefa simples) com movimentos em que após contactar o $1^{\circ}$ alvo, iniciase um novo deslocamento (tarefa complexa).

O tempo proporcional para se atingir o pico de velocidade para $\mathrm{o} 1^{\circ}$ alvo (TPPV1) foi calculado como: TPPV $1=[$ TPV X 100] $/$ TM sendo $\mathrm{TPV}=$ pico de velocidade e $\mathrm{TM}=$ tempo de movimento para o $1^{\circ}$ alvo. Esta medida relativa foi utilizada para uma melhor noção de qual momento da trajectória este evento ocorreu.
O número de correcções após o pico de velocidade para o $1^{\circ}$ alvo foi calculado através do número de descontinuidades no perfil de aceleração representadas por zero-crossings (cruzamento da curva de aceleração no eixo $\mathrm{X}$ ) e desvios significantes em aceleração e desaceleração sem mudança de sinal (mínimo de $10 \%$ do mais alto pico de aceleração durante 10 quadros). As análises foram realizadas entre o $1^{\circ}$ quadro após se atingir o pico de velocidade e o $1^{\circ}$ quadro em que o dedo indicador contactava o alvo ou suas proximidades.

Foi calculado o índice de lateralidade absoluto (IL) em termos de preferência manual e desempenho através da seguinte fórmula:

$\mathrm{IL}=[\mathrm{MD}-\mathrm{ME} / \mathrm{MD}+\mathrm{ME}] \mathrm{x} 100$

em que MD representa o resultado da média entre os sujeitos com a mão direita e ME representa o resultado da média entre os sujeitos com a mão esquerda. Os índices absolutos de assimetria lateral apontam a diferença entre as mãos em cada uma das medidas de desempenho e medidas cinemáticas independente da direcção da assimetria. Dessa forma, serão comparados os índices absolutos de assimetria lateral entre as medidas de desempenho e cinemática para possível detecção de qual medida apresenta maior índice de assimetria entre mãos. Escores próximos de zero indicam baixa assimetria entre membros.

\section{RESULTADOS}

Para cada uma das medidas de desempenho e medidas cinemáticas, foram analisadas as médias e os desvios padrão das 5 tentativas de cada tarefa com cada uma das mãos. Previamente a análise dos dados foi conduzido o teste de Kolmogorov-Smirnov, que indicou normalidade tanto para as medidas de desempenho quanto para as medidas cinemáticas. As análises inferenciais foram realizadas através de 1) Anova two-way (2 mãos X 2 níveis de complexidade) para as medidas de desempenho e cinemáticas, 2) Anova two-way (2 níveis de complexidade $\mathrm{X} 6$ blocos) para a análise do índice de lateralidade e 3 ) teste $t$ para as medidas de tempo de resposta em cada nível de complexidade. O critério de utilização do teste $t$ de Student para cada nível de complexidade partiu do princípio que a análise conjunta de tempos de resposta de tarefas com diferentes números de com- 
Tabela 1. Médias e desvio padrão das médias para as medidas de: 1] tempo de reacção (TR]; tempo de resposta na tarefa complexa (TResp TC); tempo de resposta na tarefa simples (TResp TS); tempo de contacto para o $1^{\circ}$ alvo (TCA).

\begin{tabular}{lcccc}
\hline Medidas (ms) & MD TC & Membros e complexidade da tarefa & MD TS & ME TS \\
\hline TR & $208.87 \pm 33,5$ & $211.85 \pm 33,1$ & $197.28 \pm 29,6$ & $203.73 \pm 23,6$ \\
\hline TResp TC & $705.35 \pm 67,5$ & $750.50 \pm 72$ & - & - \\
\hline TResp TS & - & - & $448.53 \pm 59,6$ & $489.32 \pm 71,7$ \\
\hline TCA & $214.72 \pm 24,48$ & $228.93 \pm 32,29$ & $201.11 \pm 39,73$ & $208.07 \pm 42,02$ \\
\hline
\end{tabular}

ponentes não seria adequado. Dessa forma, o objectivo foi o de analisar isoladamente o efeito da assimetria manual em cada nível de complexidade. Para análises Post Hoc, foi utilizado o teste de Tukey, quando analisadas mais de duas médias. $\mathrm{O}$ valor de significância adoptado foi de $\mathrm{p}<0,05$.

\section{Tempo de reação}

A análise do tempo de reacção (Tabela 1) não indicou diferença significativa entre Mãos $[\mathrm{F}(1,18)=$ $0,81, \mathrm{p}=0,77]$, assim como na interacção entre Mãos X Complexidade $[F(1,18)=0,63, p=0,80]$. Foi encontrado um efeito significativo para o factor Grau de Complexidade da Tarefa $[\mathrm{F}(1,18)=4,89$, $\mathrm{p}<$ $0,05]$ indicando um menor tempo de reacção médio para os movimentos de menor complexidade comparados aos de maior complexidade.

A análise do desvio padrão do tempo de reacção (Tabela 1) não indicou diferença significativa entre Mãos $[F(1,18)=0,22, p=0,64]$, Grau de Complexidade da Tarefa $[\mathrm{F}(1,18)=0,94, \mathrm{p}=0,34] \mathrm{e}$ interacção entre Mãos X Complexidade $[\mathrm{F}(1,18)=$ $0,16, p=0,68]$.

\section{Tempo de resposta para a tarefa complexa}

$\mathrm{Na}$ análise do tempo de resposta, o teste $t$ para medidas dependentes indicou diferença significante entre Mãos $[t(9)=-2,76, \mathrm{p}<0,05]$, tendo a mão esquerda apresentado maior tempo de resposta comparada à mão direita (Tabela 1). Não foi encontrada diferença significativa na análise do desvio padrão (Tabela 1) $[t(9)=-0,28, \mathrm{p}=0,78]$.

\section{Tempo de resposta para a tarefa simples}

$\mathrm{Na}$ análise do tempo de resposta, o teste $t$ para medidas dependentes indicou diferença significante entre Mãos $[t(9)=-2,32, \mathrm{p}<0,05]$, tendo a mão esquerda apresentado maior tempo de resposta comparada à mão direita (Tabela 1). Não foi encontrada diferença significativa na análise do desvio padrão (Tabela 1) $[t(9)=-0,60, \mathrm{p}=0,55]$.

\section{Tempo de contato para o $1^{\circ}$ alvo}

A análise do tempo de movimento para o contato do $1^{\circ}$ alvo (Tabela 1) não indicou diferença significativa entre Mãos $[F(1,18)=0,50, p=0,48]$, assim como na interação entre Mãos X Complexidade $[\mathrm{F}(1,18)=$ $0,21, p=0,64]$. Foi encontrada uma diferença significante para o fator Grau de Complexidade da Tarefa $[\mathrm{F}(1,18)=4,89, \mathrm{p}<0,05]$ indicando um menor tempo de movimento na tarefa menos complexa. A análise do desvio padrão do tempo de movimento para o $1^{\circ}$ alvo (Tabela 1 ) não indicou diferença significativa entre Mãos $[F(1,18)=0,33, p=0,85]$, Grau de Complexidade da Tarefa $[\mathrm{F}(1,18)=2,79, \mathrm{p}=$ $0,11]$ e na interação entre Mãos X Complexidade $[\mathrm{F}(1,18)=0,13, \mathrm{p}=0,71]$.

\section{Pico de velocidade para o $1^{\circ}$ alvo}

A análise do pico de velocidade não indicou diferença significativa entre Mãos $[F(1,18)=0,01, p=0,91]$, assim como na interação entre Mãos $\mathrm{X}$ Complexidade $[F(1,18)=0,01, p=0,91]$. Foi encontrada uma diferença significante para o fator Grau de Complexidade da Tarefa $[\mathrm{F}(1,18)=8,34, \mathrm{p}<0,01]$ indicando um menor pico de velocidade para os movimentos de maior complexidade $(2,14 \mathrm{~m} / \mathrm{s})$ comparados aos de menor complexidade $(2,38 \mathrm{~m} / \mathrm{s})$ (Figura 2). A análise do desvio padrão do tempo de reacção (figura 2) não indicou diferença significativa 


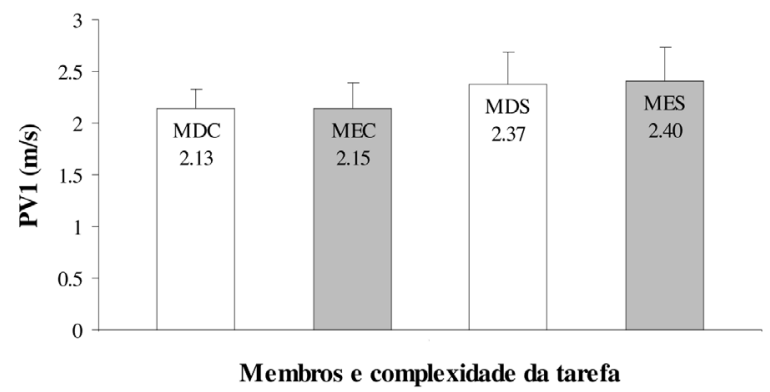

Figura 2. Pico de velocidade em direcção ao $1^{\circ}$ alvo entre membros e complexidade da tarefa $(M D C=$ mão direita tarefa complexa; $M \varepsilon C=$ mão esquerda tarefa complexa; MDS = mão direita tarefa simples; $M E S$ = mão esquerda tarefa simples].

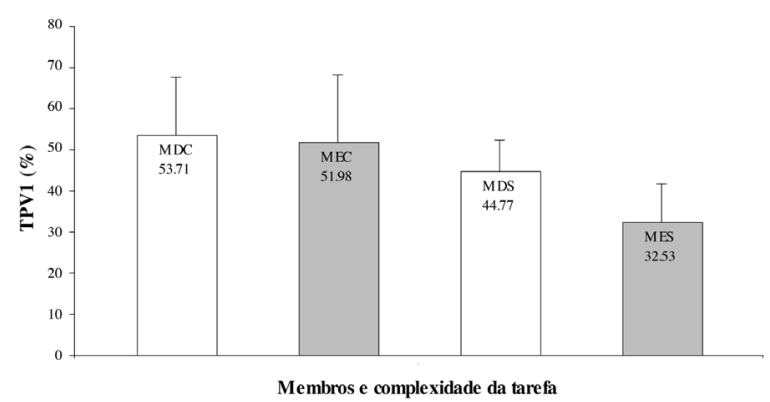

Figura 3. Tempo proporcional para se atingir o pico de velocidade entre membros e complexidade da tarefa $(M D C=$ mão direita tarefa complexa; $M E C=$ mão esquerda tarefa complexa; $M D S=$ mão direita tarefa simples; $M \varepsilon S=$ mão esquerda tarefa simples].

entre Mãos $[\mathrm{F}(1,18)=0,27, \mathrm{p}=0,60]$ e na interacção entre Mãos X Complexidade $[\mathrm{F}(1,18)=0,38, \mathrm{p}=$ $0,54]$. Foi encontrada uma diferença significante para o factor Grau de Complexidade da Tarefa $[\mathrm{F}(1,18)=11.53, \mathrm{p}<0,01]$ indicando uma menor variabilidade do movimentos complexos $(0,21 \mathrm{~m} / \mathrm{s})$ comparados aos de menor complexidade $(0,32 \mathrm{~m} / \mathrm{s})$.

\section{Tempo proporcional para se atingir} o pico de velocidade para o $1^{\circ}$ alvo

A análise do tempo para se atingir o pico de velocidade não indicou diferença significativa entre Mãos $[F(1,18)=1,24, p=0,27]$, assim como na interação entre Mãos X Complexidade $[\mathrm{F}(1,18)=1,94, \mathrm{p}=$ $0,18]$. Foi encontrada uma diferença significante para o factor Grau de Complexidade da Tarefa $[F(1,18)=13,42, p<0,05]$ indicando um menor per- centual de tempo para se atingir o pico de velocidade para o movimento de menor complexidade $(38,6 \%)$ comparado ao de maior complexidade $(52,4 \%)$

(Figura 3).

A análise do desvio padrão (Figura 3) não indicou diferença significativa entre Mãos $[F(1,18)=0,65$, $\mathrm{p}=0,42]$, Grau de Complexidade da Tarefa $[F(1,18)=2,65, p=0,12]$ e na interação entre Mãos $\mathrm{X}$ Complexidade $[\mathrm{F}(1,18)=0,01, \mathrm{p}=0,90]$.

\section{Número de correcções após o pico de velocidade para o $1^{\circ}$ alvo}

A análise do número de correcções na trajectória para o $1^{\circ}$ alvo indicou diferença significante entre Mãos $[\mathrm{F}(1,18)=4,43, \mathrm{p}<0,05]$ apontando que o membro direito efectuou um menor número de correcções $(1,3)$ comparado ao membro esquerdo $(2,1)$ (Figura 4). Não foi encontrada diferença significativa para o factor Grau de Complexidade da Tarefa $[\mathrm{F}(1,18)=$ $0,42, p=0,52]$, assim como na interacção entre Mãos $\mathrm{X}$ Complexidade $[\mathrm{F}(1,18)=1,75, \mathrm{p}=0,20]$.

A análise do desvio padrão (Figura 4) não indicou diferença significativa entre Mãos $[F(1,18)=0,20$, $\mathrm{p}=0,65]$, Grau de Complexidade da Tarefa $[F(1,18)=0,63, p=0,43]$ e na interacção entre Mãos X Complexidade $[F(1,18)=0,23, p=0,63]$.

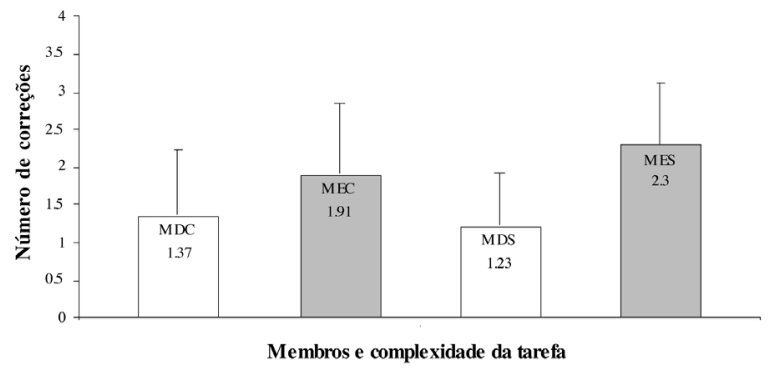

Figura 4. Número de correcções na trajectória do movimento entre membros e complexidade da tarefa $(M D C=$ mão direita tarefa complexa; $M E \mathcal{E C}=$ mão esquerda tarefa complexa; $M D S=$ mão direita tarefa simples; $M \varepsilon S=m a \tilde{o}$ esquerda tarefa simples].

\section{Índice de lateralidade}

A análise do índice de lateralidade (Figura 5) não indicou diferença significativa entre Grau de Complexidade da Tarefa $[\mathrm{F}(1,18)=0,10, \mathrm{p}=0,75]$, assim como na interacção entre Grau de Complexidade da Tarefa e Medida $[\mathrm{F}(5,90)=0,19$, 


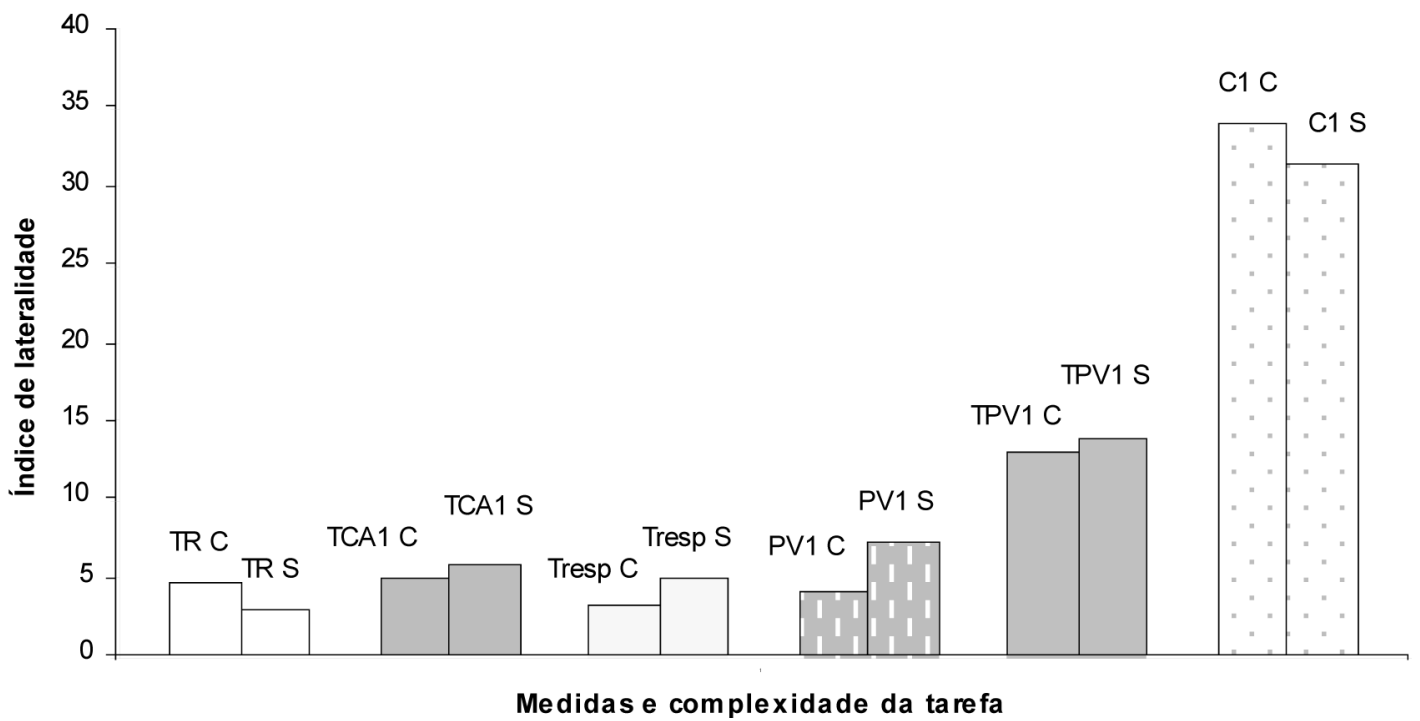

Figura 5. Índices de lateralidade entre complexidades da tarefa $\left(T R=\right.$ tempo de reacção; TCA = tempo de contacto para $01^{\circ}$ alvo alvo; Tresp = tempo de resposta; $P V 1$ = pico de velocidade; TPV1 = tempo proporcional para atingir o pico de velocidade; $C 1$ = número de correcções; a letra $C$ corresponde à tarefa complexa e a letra $S$ corresponde à tarefa simples].

$\mathrm{p}=0,96]$. Foi encontrada diferença significante para o fator Medida $[\mathrm{F}(5,90)=13,65, \mathrm{p}<0,01]$. O teste Post Hoc de Tukey indicou um maior índice de lateralidade absoluto entre as mãos para a medida de número de correcções após o pico de velocidade para o $1^{\circ}$ alvo comparado às outras medidas $(\mathrm{p}<0,01$, respectivamente).

\section{DISCUSSÃO E CONCLUSÃO}

O presente estudo teve como objectivo investigar a assimetria manual em duas tarefas de apontamento com diferentes níveis de complexidade. Para tal, foram analisadas medidas de desempenho e medidas cinemáticas para inferências sobre as possíveis diferenças no controle de ambas as mãos em uma tarefa de menor complexidade e de maior complexidade. Os resultados apresentam alguns indicativos de assimetria, tanto no desempenho como na análise cinemática. Em relação à assimetria no tempo de preparação do movimento, não foi observada vantagem do sistema hemisfério direito/mão esquerda em relação ao sistema contralateral. Este é um resultado de certa forma previsível devido a resultados contraditórios encontrados na literatura ${ }^{(5,6)}$. De forma geral, assume-se que o sistema hemisfério direito/mão esquerda é especializado na parametrização espacial do movimento(5). Entretanto, este não parece ser um fenómeno robusto, parecendo haver somente uma tendência de a mão esquerda reagir mais rapidamente do que a mão direita(6). Resultados similares em tarefas que exigem tempo de reação simples, como o do presente estudo, têm sido encontrados(18). A medida de tempo de resposta mostrou maior velocidade do membro direito comparado ao esquerdo em destros, fenómeno robusto nas assimetrias manuais (2, 6), e explicado pela especialização do sistema hemisfério esquerdo/mão direita no controle seriado e processamento sequencial dos movimentos. Apesar de não terem sido encontradas diferenças significativas entre as mãos para as medidas de tempo de reacção e tempo de contacto para o $1^{\circ}$ alvo, os resultados mostram que a soma destes dois momentos que compõem o tempo de resposta contribui para a assimetria manual no tempo de resposta.

A análise cinemática mostrou que o número de correcções para atingir o alvo, em ambos os níveis de complexidade, foi menor na mão direita que na esquerda. Tais resultados mostram a maior capacidade do sistema hemisfério esquerdo/mão direita em lidar com o processamento on-line, corroborando pré- 
vios achados de pesquisa ${ }^{(6,12)}$. A ausência de assimetria para os valores de pico de velocidade, indica que não houve diferenças na especificação de força para o deslocamento dos membros em direcção ao alvo, mas sim, que as assimetrias na execução do movimento estão relacionadas a processos correctivos envolvidos na segunda fase do movimento. Essa hipótese é consistente com os resultados encontrados sobre os índices de lateralidade. Os índices mais altos de diferença entre membros foram encontrados nas medidas do número de correcção tanto na tarefa complexa, quanto na tarefa simples. Mesmo não tendo sido objectivo de investigação do presente estudo, é possível inferir que esse menor número de correcções apresentado pelo membro direito na fase de controle corrente é também um indicador de ajustes on-line mais eficazes que levam a uma maior precisão.

Em relação à complexidade da tarefa, de forma geral os resultados do presente estudo não suportam os prévios achados de pesquisas $(7,8,14,15)$. A análise do desempenho de ambos os membros nas duas tarefas não mostrou um efeito da complexidade sobre as assimetrias manuais, contrariando assim, tanto os resultados que apontam para uma maior assimetria manual em tarefas mais complexas $(7,15)$, quanto os resultados que assinalam para uma maior assimetria em tarefas menos complexas $(8,14)$. Como a definição de complexidade da tarefa depende da relativização do que é comparado, é necessário enfatizar que em tarefas de apontamento com diferentes números de componentes não foram encontrados efeitos de assimetria manual. A maioria das diferenças encontradas se refere ao efeito da complexidade sobre as características de desempenho e cinemáticas das tarefas. Este efeito foi encontrado nas medidas de tempo de reacção, tempo de contacto para o $1^{\circ}$ alvo, pico de velocidade para o $1^{\circ}$ alvo e tempo proporcional para se atingir o pico de velocidade para o $1^{\circ}$ alvo. Tais resultados dão suporte a nossa proposta de que as tarefas foram distintas em termos de complexidade. A tarefa mais complexa apresentou maior tempo de reacção comparada à tarefa menos complexa. Esse resultado era esperado, na medida em que movimentos mais complexos requerem maior demanda na programação motora e, consequentemente, maior tempo de processamento(10,18). A análise do tempo de movimento para contacto do $1^{\circ}$ alvo também indicou um efeito similar ao do tempo de reacção. O movimento mais complexo despendeu maior tempo comparado ao movimento de menor complexidade, efeito definido como OTA(1). Uma das possíveis explicações sobre este efeito da complexidade da tarefa sobre o tempo de movimento se baseia na visão que sub-rotinas são armazenadas em um buffer na tarefa com mais de um componente. À medida que a primeira sub-rotina dá início à primeira parte do movimento, deslocando o membro em direcção ao $1^{\circ}$ alvo, uma segunda sub-rotina é implementada gerando um maior custo no controle motor devido ao processamento paralelo(1). Os resultados do presente estudo reforçam os achados de um estudo que investigou o OTA nas execuções do membro esquerdo(11), como também mostram este robusto efeito em movimentos realizado em um espaço de trabalho do membro esquerdo diferente ao anteriormente investigado(11), tendo em vista que esta variável interfere no controle motor( ${ }^{(12)}$.

A análise dos perfis de velocidade e aceleração auxilia no entendimento dos processos envolvidos na execução da tarefa de apontamento. A menor magnitude e variabilidade do pico de velocidade apresentada na tarefa de maior complexidade, assim como o maior tempo para se atingir o pico e velocidade, suportam as predições sobre a maior restrição sofrida no controle quando um segundo movimento é requerido(11). Isto porque a implementação do $2^{\circ}$ movimento que ocorre durante a execução do $1^{\circ}$ movimento reflecte em movimentos mais controlados em termos de força na fase de impulso inicial e com maior exigência de tempo para que o pico de velocidade seja alcançado. Esses processos envolvidos são de natureza tanto central, na preparação do movimento, quanto no controle corrente durante o movimento(11).

Como as diferenças apresentadas pelos membros foram identificadas em ambas as tarefas, não foi encontrado o efeito da complexidade sobre as assimetrias manuais. Os estudos que apontam para uma maior assimetria em tarefas de menor complexida-

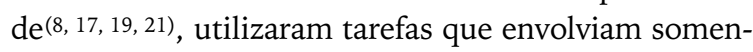
te o controle das falanges dos dedos. Por outro lado, os estudos que mostram uma maior assimetria em tarefas de maior complexidade $(7,15)$ utilizaram tarefas 
de diferentes naturezas. Os resultados do presente estudo sugerem uma especificidade das variáveis a serem estudadas. No presente estudo o comportamento gerado pelos membros nas tarefas de menor e maior complexidade foi diferente em termos de programação motora. Entretanto, é possível que processos excitatórios e/ou inibitórios envolvidos na activação dos hemisférios cerebrais tenham sido similares nessas tarefas o que geraria um mesmo nível de assimetria manual em ambas as tarefas. Neste caso, a limitação sobre o que é complexidade da tarefa novamente emerge, podendo ser que a variável "número de componentes" em tarefas de apontamento não seja um factor de influência na relação assimetrias manuais e complexidade da tarefa.

Uma sugestão para próximos estudos é a utilização de medidas comportamentais para a definição de lateralidade dos participantes ao invés de utilização de inventário de preferência manual. A adopção da análise de desempenho em diferentes tarefas manuais pode garantir uma forma mais eficiente de medida da lateralidade da amostra (2). Uma segunda sugestão é a investigação da relação entre (1) os processos de correcção on-line, (2) precisão do movimento e (3) assimetrias manuais. Seria o menor número de correcções apresentado pelo membro direito na fase de controle corrente um indicador de ajustes on-line mais eficazes que levam a uma maior precisão? E por fim, sugerem-se novos estudos que investiguem os efeitos da complexidade da tarefa na assimetria manual em movimentos realizados no espaço de trabalho contralateral ao do membro de execução. Isto porque diferentes níveis de activação hemisférica podem ser gerados nesta condição, trazendo novos conhecimentos sobre as variáveis envolvidas nas assimetrias manuais.

\section{CORRESPONDÊNCIA}

\section{Guilherme Lage}

Universidade FUMEC / FCS, Faculdade de Ciências

da Saúde

Departamento de Educação Física

Rua da Paisagem, 240 - Vila da Serra

Nova Lima - Minas Gerais, Brasil

CEP 34000-000

E-mail: menezeslage@gmail.com 


\section{REFERÊNCIAS}

1. Adam JJ, Nieuwenstein JH, Huys R, Paas FGW, Kingma H, Willems P, Werry M (2000). Control of rapid aimed hand movements: the one-target advantage. Journal of Experimental Psychology: Human Perception and Performance 26: 295-312.

2. Bryden PJ (2000). Lateral preferences, skilled behavior and task complexity: Hand and foot. In: Mandal MK, BulmaFleming MB, Tiwari G (Eds.). Side bias: a neuropsychological perspective. Dordrecht: Kluwer Academic Publishers.

3. Buekers MJ, Helsen WF (2000). Vision and laterality: does occlusion disclose a feedback processing advantage for the right hand system? Cortex 36: 507-519.

4. Carson RG (1992). Visual feedback processing and manual asymmetries: an evolving perspective. In: Proteau L, Elliott D (eds.). Vision and Motor Control, Amsterdam: NorthHolland, p. 49-65.

5. Carson RG (1996). Putative right hemisphere contributions to the preparation of reaching and aiming movements. In: Elliott D, Roy EA (Eds.). Manual asymmetries in motor performance. Boca Raton: CRC.

6. Elliott D, Chua R (1996). Manual asymmetries in goaldirected movement. In: Elliott D, Roy EA (Eds.). Manual asymmetries in motor performance. Boca Raton: CRC.

7. Flowers K (1975). Handedness and controlled movement. British Journal of Psychology, 66: 39.

8. Hausmann M, Kirk LJ, Corballis PM (2004). Influence of task complexity on manual asymmetries. Cortex 40: 103110.

9. Henry FM, Rogers DE. (1960). Increased response latency for complicated movements and a "Memory Drum" theory of neuromotor reaction. Research Quarterly for Exercise and Sport, 31: 448-458.

10. Khan MA, Franks IM (2003). Online versus offline processing of visual feedback in the production of component submovements. Journal of Motor Behavior 35: 285-295.

11. Lavrysen A, Helsen WF, Tremblay L, Elliott D, Adam JJ, Feys P, Buekers MJ (2003). The control of sequential aiming movements: the influence of practice and manual asymmetries on the one-target advantage. Cortex 39: $307-$ 325.

12. Mieschke PE, Elliott D, Helsen WE, Carson RG, Coull JA (2001). Manual asymmetries in the preparation of goaldirected movements. Brain and Cognition 45: 129-140.

13. Oldfield RC (1971). The assessment and analysis of handedness: the Edinburgh inventory. Neuropsychologia 9: 97113.

14. Passarotti AM, Banich MT, Sood RK, Wang JM (2002). A generalized role of interhemispheric interaction under attention ally demanding conditions: evidence from the auditory and tactile modality. Neuropsychologia 40: 10821096.

15. Provins KA, Magliaro J (1993). The measurement of handedness by preference and performance tests. Brain and Cognition 22: 171-181.

16. Santos IM, Lage GM, Calvacante AP, Ugrinowitsch H, Benda RN (2006). Análise da assimetria nos padrões fundamentais arremessar e chutar em crianças. Revista Portuguesa de Ciências do Desporto 6: 188-193.

17. Solodkin A, Hlustik P, Noll DC, Small SL (2001). Lateralization of motor circuits and handedness during finger movements. European Journal of Neurology 8: 425-434.
18. Teixeira LA, Gasparetto RE, Sugie MM (1999). Is there manual asymmetry in movement preparation? Perceptual and motor skills 89: 205-208.

18. Tinazzi M, Zanette G (1998). Modulation of ipsilateral motor cortex in man during unimanual finger movements of different complexities. Neuroscience Letters 224: 121-124.

19. Velay J, Benoit-Dubrocard S. (1999). Hemispheric asymmetry and interhemispheric transfer in reaching programming. Neuropsychologia. 37:895-903.

20. Verstynen T, Diedrichsen J, Albert N, Aparicio P, Ivry RB (2004). Ipsilateral motor cortex activity during unimanual hand movements relates to task complexity. Journal of Neurophysiology 93: 1209-1222.

21. Woodworh RS (1899). The accuracy of voluntary movement. Psychological Review 3 (suplemento 2). 